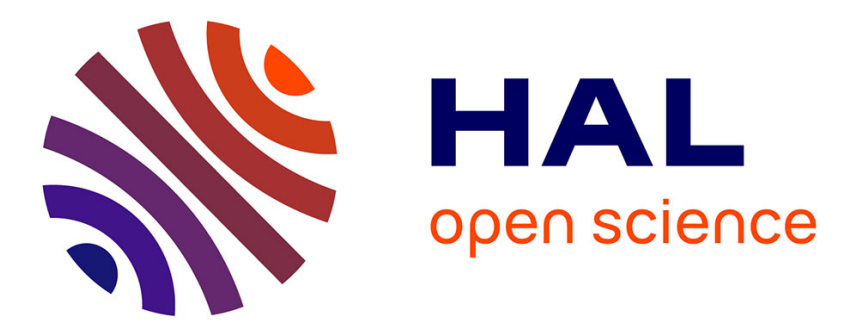

\title{
Circularity measuring in linear time
}

Thanh Phuong Nguyen, Isabelle Debled-Rennesson

\section{To cite this version:}

Thanh Phuong Nguyen, Isabelle Debled-Rennesson. Circularity measuring in linear time. 20th International Conference on Pattern Recognition - ICPR 2010, Aug 2010, Isatanbul, Turkey. pp.2098-2101. inria-00544736

\section{HAL Id: inria-00544736 \\ https://hal.inria.fr/inria-00544736}

Submitted on 10 Dec 2010

HAL is a multi-disciplinary open access archive for the deposit and dissemination of scientific research documents, whether they are published or not. The documents may come from teaching and research institutions in France or abroad, or from public or private research centers.
L'archive ouverte pluridisciplinaire HAL, est destinée au dépôt et à la diffusion de documents scientifiques de niveau recherche, publiés ou non, émanant des établissements d'enseignement et de recherche français ou étrangers, des laboratoires publics ou privés. 


\title{
Circularity measuring in linear time*
}

\author{
Thanh Phuong NGUYEN and Isabelle DEBLED-RENNESSON \\ LORIA Nancy, Campus Scientifique - BP 23954506 Vandøuvre-lès-Nancy Cedex, France \\ $\{$ nguyentp,debled $\}$ loria.fr
}

\begin{abstract}
We propose a new circularity measure inspired from Arkin [1], Latecki [9] tools of shape matching that is constructed in a tangent space. We then introduce a linear algorithm that uses this measure for circularity measuring. This method can also be regarded as a method for circular object recognition. Experimental results show the robustness of this simple method.
\end{abstract}

\section{Introduction}

Digital circle is a basic object that is an interesting topic in image analysis and discrete geometry. In the literature, there are two well-known problems concerning digital circles: circle recognition and circularity measuring. The first problem decides if a digital curve is a digitization of a circle. The second one decides how far a digital curve is from a digitization of a circle.

Many methods for circle recognition have been proposed. Kim [7] detects if a set of grid points in a $N \mathrm{x}$ $N$ image is a digital disk with a complexity in $O\left(n^{3}\right)$. Later, he [8] reduced this complexity to $O\left(n^{2}\right)$. Coeurjolly [2] transformed the problem of circle recognition into the search of a $2 \mathrm{D}$ point that belongs to the intersection of $n^{2}$ half-planes. It can be solved in $O\left(n^{4 / 3} \log n\right)$ time. Sauer [14] (resp. Damaschke [3]) used a sophisticated tool in linear programming [11] to propose a linear method for circle (resp. arc) recognition.

Beside the problem of circle recognition, circularity measure (or compactness measure) is also an interesting problem that was presented firstly by Rosenfeld [12]. He proposed the square of the perimeter of a figure divided by its area, $P^{2} / A$, to measure the non-compactness of the figure. Haralick [6] introduced four principal properties to construct a good measure for the circularity of simple closed figures. Later, Kim [8] presented a new circularity measure for convex digital regions. It is constructed from his algorithm of disk segmentation. Considering a convex region $P, P_{0}$ denotes the smallest disk containing $P, A_{P}$ (resp. $A_{P_{0}}$ ) denotes the areas of $P$ (resp. $\left.P_{0}\right)$. He introduced $m_{p}=A_{P} / A_{P_{0}}$ as compactness measure. An algorithm

\footnotetext{
* This work is supported by ANR in the framework of GEODIB project, BLAN06-2 134999.
}

that uses this measure was also introduced in $O\left(n^{3} \sqrt{n}\right)$. Roussillon [13] gave a new circularity measure for digital curves based on the separating circle problem. From a digital 4-connected curve, he constructed two finite sets of points $S$ and $T$ by regarding the interior points of this curve. To verify whether $\mathrm{S}$ and $\mathrm{T}$ are separable by a circle, he transformed them into $S^{\prime}$ and $T^{\prime}$ in the dual space. The minimum height between the two convex hulls $C H\left(S^{\prime}\right)$ and $C H\left(T^{\prime}\right)$ was given as a circularity measure. Based on this measure, an algorithm for circularity measuring [13] is proposed in $O(n \log n)$.

In this paper, we present a new circularity measure for a digital curve. We will show that it satisfies the four principal Haralick's properties to be a good circularity measure. We also introduce a linear algorithm for circularity measuring of closed curves based on this measure. The rest of this paper is organized as follows. The next section recalls tangent space representation and tools of similarity measure related to this space. Section 3 mentions the representation of real circles in the tangent space. Section 4 proposes a new circularity measure and presents a linear algorithm for circularity measuring based on this measure. The last section introduces some experimentations and a conclusion.

\section{Tangent space and shape matching}

Latecki proposed a space, named tangent space, to represent polygonal curves [9]. By using this representation, a similarity measure was also proposed in this work as a tool of shape matching. They are recalled in this section.

\subsection{Tangent space representation}

Let $\zeta=\left\{C_{i}\right\}_{i=0}^{n}$ be a polygonal curve, $\alpha_{i}=$ $\angle\left(\overrightarrow{C_{i-1} C_{i}}, \overrightarrow{C_{i} C_{i+1}}\right)$ and $l_{i}$ - the length of the segment $\overrightarrow{C_{i} C_{i+1}}, i \in\{0, \ldots, n-1\}$. We have $\alpha_{i}>0$ if $C_{i+1}$ is on the right of $\overrightarrow{C_{i-1} C_{i}}, \alpha_{i}<0$ otherwise.

$L=\sum_{i=0}^{n-1} l_{i}, l_{i}^{\prime}=\frac{l_{i}}{L}, i \in\{0, \ldots, n-1\}$

We consider the transformation that associates a polygon $\zeta$ of $\mathbb{Z}^{2}$ in a polygon of $\mathbb{R}^{2}$ that is constituted by the segments $T_{i 2} T_{(i+1) 1}, T_{(i+1) 1} T_{(i+1) 2}$ for $i \in\{0, \ldots, n-1\}$ with: 
$T_{02}=(0,0)$,
$T_{i 1}=\left(T_{(i-1) 2} \cdot x+l_{i-1}^{\prime}, T_{(i-1) 2} \cdot y\right), i \in\{1, \ldots, n\}$,
$T_{i 2}=\left(T_{i 1} \cdot x, T_{i 1} \cdot y+\alpha_{i}\right), i \in\{1, \ldots, n-1\}(n$ if $\zeta$ is a closed curve, $C_{0} \equiv C_{n}$ ).

Fig. 1 illustrates how to transform a polygonal curve into the tangent space representation.

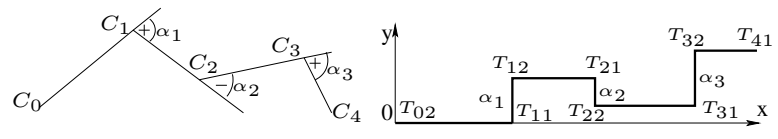

(a) Input polygon

(b) Its tangent space representation

Figure 1. Transform of tangent space

Remark 1 If $\zeta$ is a closed convex curve (in clockwise direction), the coordinate of $T_{n 1}$ is $(1,2 \pi)$.

This remark comes from the fact that the sum of all interior angles in a $n$-convex polygon is $(n-2) \pi$.

\subsection{Similarity of polygonal shapes}

Arkin et al. proposed a method based on $L_{2}$ distance [1] as a metric for comparing polygonal shapes. Later, Latecki et al. [9] defined shape similarity measure related to the one of Arkin et al.

Let c, $\mathrm{d}$ be simple polygons; $T(c), T(d)$ be their tangent functions. The similarity measure $[9,10]$ between both polygons is given by

$$
\begin{gathered}
S_{a}(c, d)=\left(\int_{0}^{1}\left(T(c)(s)-T(d)(s)+\theta_{0}\right)^{2} d s\right) \times \\
\max (l(c), l(d)) \max \left(\frac{l(c)}{l(d)}, \frac{l(d)}{l(c)}\right)
\end{gathered}
$$

where $l$ is the arc-length of the corresponding polygon. The integral in the above equation is weighted with the arc length penalized by the difference in length of the corresponding parts. The constant $\theta_{0}$ is a translation of $T(d)$ that minimizes the integral $\int_{0}^{1}(T(c)(s)-$ $\left.T(d)(s)+\theta_{0}\right)^{2} d s$. It is given by lemma 3 in [1].

\section{Circle in the tangent space}

Without loss of generality, we suppose that a real circle is considered in the clockwise direction, we then propose the theorem below.

Theorem 1 A real circle is represented in the tangent space by the straight line $O E,(O(0,0), E=(1,2 \pi))$.

Proof 1 Let us study the tangent space representation $T(\zeta)$ of a $n$-sided convex regular polygon $\zeta=\left\{C_{i}\right\}_{i=0}^{n}$ (clockwise direction) that is inscribed in a circle of radius $R$ noted as $\zeta(R)$ (see Fig. 2.a). Fig. 2.b shows the

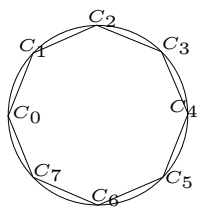

(a) A regular polygon

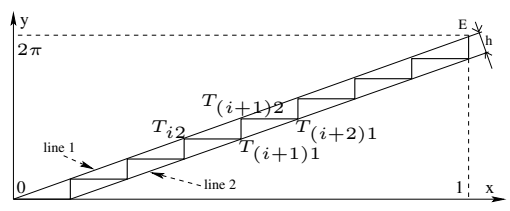

(b) Representation of this polygon in tangent space

\section{Figure 2. A regular polygon and its cir- cumcircle.}

tangent space representation of the regular polygon and its circumcircle.

Considering the two points at the extremities of $T(\zeta): T_{02} \equiv O(0,0), T_{n 2} \equiv E(1,2 \pi)$. Thanks to the regular property of this polygon, the length at each side, and the angle at each vertex of it are the same. Therefore, in the corresponding tangent space representation of this polygon $T(\zeta)$ (see Fig. 2.b), we have: $T_{02} T_{11}=\ldots=T_{(i-1) 2} T_{i 1}=\ldots=T_{(n-1) 2} T_{n 1}$, and $T_{11} T_{12}=\ldots=T_{i 1} T_{i 2}=\ldots=T_{(n-1) 1} T_{(n-1) 2}$. Thanks to Thales' theorem, $T 1=\left\{T_{(i+1) 1}\right\}$ and $T 2=$ $\left\{T_{i 2}\right\}$ are two sets of colinear points. We consider the two real lines: line 1, and line 2 that contain these two sets (see Fig. 2.b). Let $h$ be the perpendicular distance between line 1 and line 2 . It is trivial that $0 \leq h<T_{i 1} T_{i 2}=\frac{2 \pi}{n}, i \in\{1, \ldots, n-1\}$. Thanks to $\lim _{n \rightarrow+\infty} \frac{2 \pi}{n}=0$, we obtain $\lim _{n \rightarrow+\infty} h=0$. In addition, line 1 always passes through the two points $O(0,0)$ and $E(1,2 \pi)$ thanks to remark 1 . It means that when $n \rightarrow+\infty$ line 1 and line 2 become the unique line that passes through $O(0,0)$ and $E(1,2 \pi)$. Because $T(\zeta)$ is between line 1 and line 2 , so when $n \rightarrow+\infty$, we have: $T(\zeta) \rightarrow O E$.

Therefore, we have this corollary below.

Corollary 1 The tangent space function of a real circle is $T(s)=2 \pi s, s \in[0,1]$

\section{Circularity measuring}

\subsection{Circularity measure}

\subsubsection{How is a good circularity measure?}

In the pioneer work [6] on circularity measuring, Haralick defined some main properties of a good measure for the circularity of a digital figure.

1. The more a figure becomes circular, the more the measure of its circularity increases.

2 . The values for digital figures follow the values for the corresponding continuous figures.

3 . It is orientation independent.

4. It is area independent.

As the existing circularity measures don't give the same range of value, we change property 1 as follows: "The more a figure becomes circular, the more the circularity measure approaches the actual value". 


\subsubsection{Proposed circularity measure}

We propose a novel circularity measure by using the similarity measure of polygons given by Latecki et al. [9]. The main idea is to determine the similarity between an input discrete curve $\zeta$ and a real circle. Thanks to Corollary 1 , the tangent space representation of a real circle is determined. Our circularity measure is proposed as follows.

$$
C M_{\zeta}=\int_{0}^{1}\left(T(\zeta)(s)-2 \pi s+\theta_{0}\right)^{2} d s
$$

where $\theta_{0}$ is defined by lemma 3 in [1] as follows.

$$
\theta_{0}=\int_{0}^{1} T(\zeta)(s) d s-\pi
$$

Because the tangent representations of all real circles are the same thanks to Theorem 1, and in addition, we want to measure the similarity between the input curve and any real circle, so we remove in equation (1) the difference of length of the corresponding parts [9].

\subsubsection{Invariant properties}

We will show that our measure fulfills the necessary invariant properties that was proposed by Haralick for circularity measure (see section 4.1.1).

1. $C M_{\zeta}$ is a metric $[1,9]$ based on $L_{2}$ distance. So, when $\zeta$ becomes circular, $C M_{\zeta} \rightarrow 0$.

2 . The second Haralick's property is satisfied because $C M_{\zeta}$ measure the similarity between $\zeta$ and a real circle, thanks to Corollary 1.

3 . If $\zeta$ is considered in the counter-clockwise direction, we consider the representation of a real circle in the tangent space as $T(s)=-2 \pi s$. So, the third Haralick's property is satisfied.

4. Thank to theorem 1 , all real circles have the same representation in the tangent space. So, $C M_{\zeta}$ is area independent.

\subsection{Proposed algorithm}

Algorithm: Based on the above theoretical study, we propose in Algorithm 1 a method for measuring the circularity of a closed curve. We suggest to work indirectly on a approximated polygon to reduce distorsion effect on similarity metric but it isn't an obligatory step.

Complexity: The transformation of a polygonal curve into the tangent space is done in linear time. It is evident that $\theta_{0}$ is determined in linear time.

$\theta_{0}+\pi=\sum_{i=0}^{n-1} \int_{T_{i 2} \cdot x}^{T_{(i+1) 2} \cdot x}\left(T_{i 2} \cdot y\right) d s=\left.\sum_{i=0}^{n-1}\left(T_{i 2} \cdot y\right) s\right|_{T_{i 2} \cdot x} ^{T_{(i+1) 2} \cdot x}$

On the other hand, $C M_{\zeta}$ can be also determined in linear time based on the notion of critical events presented in [1]. Let us suppose that $T(P)$ is represented by the sequence of segments $T_{i 2} T_{(i+1) 1}, T_{(i+1) 1} T_{(i+1) 2}$. So, $C M_{\zeta}=$

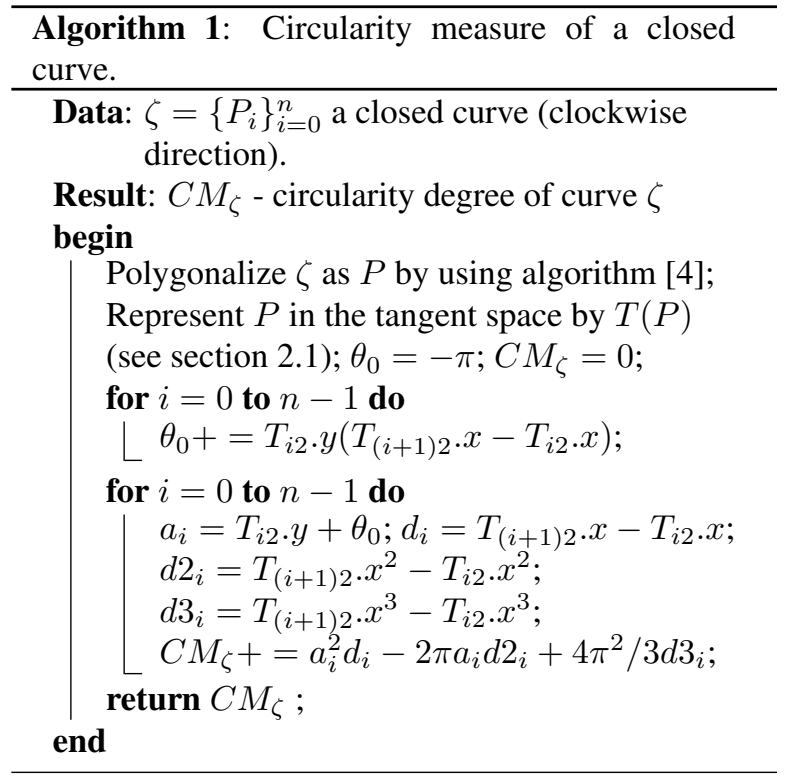

$\sum_{i=0}^{n-1} \int_{T_{i 2} . x}^{T_{(i+1) 2} \cdot x}\left(T_{i 2} \cdot y+\theta_{0}-2 \pi s\right)^{2} d s=\sum_{i=0}^{n-1}\left(\left(a_{i}^{2} s-\right.\right.$ $\left.\left.2 \pi a_{i} s^{2}+4 \pi^{2} / 3 s^{3}\right)\left.\right|_{T_{i 2} \cdot x} ^{T_{(i+1) 2} \cdot x}\right)$ where $a_{i}=T_{i 2} \cdot y+$ $\theta_{0}, i \in\{0, \ldots, n-1\}$.

So, our algorithm is executed in linear time.

\section{Experimentation}

\subsection{Experimental results}

We have developed this robust method. Figs. 3 and 4, Tab. 1 show an experimentation on fruit images and on non-circle objects.

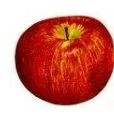

(a)

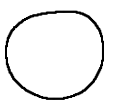

(g)

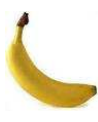

(b)

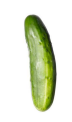

(c)

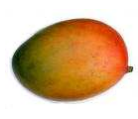

(d)

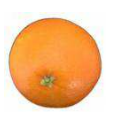

(e)

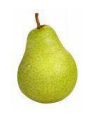

(f)

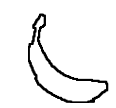

(h)

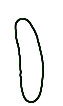

(i)

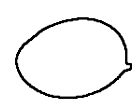

(j)

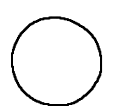

(k)

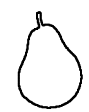

(1)
Figure 3. Fruit images and their contours.

\begin{tabular}{|c|c|c|c|c|c|c|}
\hline Fig. & $\mathrm{a}$ & $\mathrm{b}$ & $\mathrm{c}$ & $\mathrm{d}$ & $\mathrm{e}$ & $\mathrm{f}$ \\
\hline Fig. 3 & 0.055 & 15.385 & 1.591 & 0.122 & 0.076 & 7.422 \\
\hline Fig. 4 & 4.622 & 4.078 & 5.92 & 7.203 & 10.22 & \\
\hline
\end{tabular}

Table 1. Circularity measure on Figs. 3, 4. 


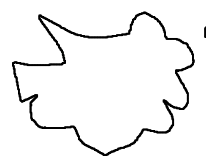

(a)

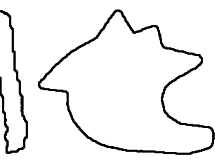

(b)

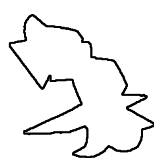

(d)

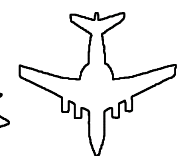

(e)

Figure 4. Test on non-circle objects

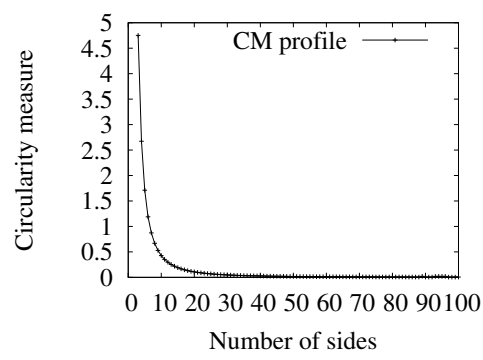

Figure 5. Test on a regular polygon set.

\subsection{Application to circular object recognition}

We note that this method can also be regarded as a method of circular object recognition if we use a threshold on the obtained result. So, this method can also recognize a circular object in linear time. Fig. 5 presents profiles of circularity measure on a set of 98 regular polygons whose the number of sides varies from 3 to 100. Fig. 6 shows a profile of circularity measure obtained on different discrete circles whose radius varies from 150 to 250. According to Tab. 1, Figs. 5, 6, we choose 0.08 as a threshold for circular object recognition in which an example is presented in Fig. 7.

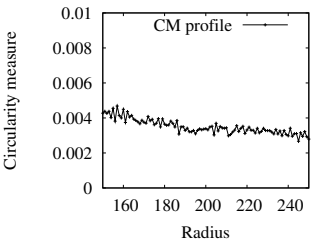

Figure 6. CM profile on different circles

\subsection{Comparison with existing methods}

The proposed method of circularity measure is simple to implement. Its linear complexity is better than Kim et al.'s method [8] $\left(O\left(n^{3} \sqrt{n}\right)\right)$, and than Roussillon et al.'s method [13] $(O(n \log n))$.

Concerning the circle recognition problem, in the literature there are only two linear methods proposed by
Sauer [14] and Damaschke [3]. These 2 methods used a sophisticated tool in linear programming [11]. So, in the contexte of circular object recognition, the proposed method is better than the others: Kim [7] $\left(O\left(n^{3}\right)\right)$, Kim [8] $\left(O\left(n^{2}\right)\right)$, Coeurjolly [2] $\left(O\left(n^{4 / 3} \log n\right)\right)$, Fisk [5] $\left(O\left(n^{2}\right)\right), \ldots$

\section{Conclusion}

We have presented a new circularity measure that fulfils the necessary properties of Haralick [6]. A linear algorithm, based on this measure, is also proposed to measure the circularity of closed curves. In addition, it is simple and robust to implement. Of course, its efficiency depends on the efficiency of the similarity measure proposed by Latecki.

\section{References}

[1] E. M. Arkin, L. P. Chew, D. P. Huttenlocher, K. Kedem, and J. S. B. Mitchell. An efficiently computable metric for comparing polygonal shapes. PAMI, 13(3):209-216, 1991.

[2] D. Coeurjolly, Y. Gérard, J.-P. Reveillès, and L. Tougne. An elementary algorithm for digital arc segmentation. Discrete Applied Mathematics, 139(1-3):31-50, 2004.

[3] P. Damaschke. The linear time recognition of digital arcs. Pattern Recognition Letters, 16(5):543-548, 1995.

[4] I. Debled-Rennesson, F. Feschet, and J. Rouyer-Degli. Optimal blurred segments decomposition of noisy shapes in linear time. Computers \& Graphics, 30(1), 2006.

[5] S. Fisk. Separating point sets by circles, and the recognition of digital disks. PAMI, 8(4):554-556, July 1986.

[6] R. Haralick. A measure for circularity of digital figures. IEEE Trans. Systems, Man, and Cybernetics, 4, 1974.

[7] C. E. Kim. Digital disks. PAMI, 6(3):372-374, May 1984.

[8] C. E. Kim and T. A. Anderson. Digital disks and a digital compactness measure. In STOC, pages 117-124, New York, NY, USA, 1984. ACM.

[9] L. Latecki and R. Lakamper. Shape similarity measure based on correspondence of visual parts. PAMI, 22(10):1185-1190, Oct 2000.

[10] L. J. Latecki and R. Lakämper. Application of planar shape comparison to object retrieval in image databases. Pattern Recognition, 35(1):15-29, 2002.

[11] N. Megiddo. Linear programming in linear time when the dimension is fixed. J. ACM, 31(1):114-127, 1984.

[12] A. Rosenfeld. Compact figures in digital pictures. IEEE Transactions on Systems, Man and Cybernetics, SMC4:221-223, 1974.

[13] T. Roussillon, I. Sivignon, and L. Tougne. Test and measure of circularity for digital curves. In IPCV'08, July 2008.

[14] P. Sauer. On the recognition of digital circles in linear time. Comput. Geom. Theory Appl., 2(5):287-302, 1993. 